\title{
KNOWLEDGE MANAGEMENT BASED APPROACH FOR VIRTUAL ORGANIZATION INHERITANCE
}

\author{
Leandro Loss, Alexandra A. Pereira-Klen, Ricardo J. Rabelo \\ Federal University of Santa Catarina, Department of Automation and Systems \\ GSIGMA - Intelligent Manufacturing Systems Group \\ Florianópolis (SC), BRAZIL \\ \{loss,klen\}@gsigma.ufsc.br; rabelo@das.ufsc.br
}

\begin{abstract}
This paper presents a framework for supporting virtual organization (VO) inheritance relying on knowledge management approach. It emphasizes that part of the problems to manage VOs is derived from the lack of information and lessons learned about the past businesses as well as that keeping the VOs' histories can be useful for augmenting and for given more agility in the decision-making process. The suggested framework presents VO inheritance as a horizontal activity that covers the entire VO life cycle, providing information for the several involved collaborative actors. An initial experiment using decision analysis is presented to show its use in vo inheritance. In the conclusions it is argued that VOs could be much more effective and the problems solved with more agility if a better use could be made out of the information inherited from VOs.
\end{abstract}

\section{INTRODUCTION}

Working collaboratively is becoming more and more important for enterprises as a strategic answer to respond to the increasing dynamics and competitiveness in the global market. Collaborative Networked Organizations (CNOs) has been considered the discipline in charge of studying all the manifestations of organizations when they work in an inter-linked and organized way (Camarinha-Matos et al., 2004a).

One of these manifestations is Virtual Organization (VO). A VO is a dynamic, temporary and logical aggregation of autonomous organizations that cooperate with each other as a strategic answer to attend a given business opportunity or to cope with a specific need, and whose operation is achieved by a coordinated sharing of skills, resources and information, totally enabled by computer networks (Rabelo et al., 2004). Its objective is to create and to deliver products or services to final customers and, at the same time, creating value for all entities engaged in the collaboration process (ECOLEAD, 2005a).

Managing the creation and the operation of VOs is, however, complex. Part of this complexity has been identified in several works, e.g. (McKenzie, 2001), (Afsarmanesh et al., 2005), (Karvonen et al., 2005). Another part of it is related to the lack of adequate support for VO managers to consider past experiences when dealing with problems along the VO life cycle. The inability to learn from a cumulative "project history" probably puts a new project's timetable in greater risk 
than all other factors combined (Reed, 2002). The same situation may occur for VOs. VOs operating without considering what have happened in past VOs will not necessarily fail, but accessing VOs' past information can require considerable - and unnecessary - effort from VO managers and from VO partners. This kind of information can be very much useful in several situations. For example, for speeding up the selection of partners for future VOs (reducing the search and negotiation processes) or for elucidating similar problems already solved in other VOs.

This work intends to exploit the part related to the difficulties originated by the fact that most of the information and knowledge generated along the VO life cycle use to be lost after the VO dissolution, i.e. the fact that VOs' history are not retained for future use.

VO inheritance (VO-I) has been emerging as the research area for dealing with the problems related to how generated information, knowledge, devised practices, products and services about VOs can be handled and managed (Camarinha-Matos et al., 2004b). In fact, this area is still in its infancy. Initial steps have been made in the ECOLEAD project (ECOLEAD, 2005c), comprising the VO creation phase, and calling bag of assets as the repository that contains common ontologies, basic software tools, and common information related to Virtual Organization Breeding Enviroments (VBE).

The proposed approach aims at presenting a framework based on knowledge management that extends the VO inheritance scope to the whole VO life cycle.

The paper is organized as follows: Section 2 introduces the concept related to VO inheritance and how it is placed in the VBE/VO context. Section 3 presents the knowledge management approach for VO inheritance. Section 4 depicts the basic concepts of decision support as well as of structuring decisions via decision analysis. Section 5 shows a practical example using decision trees and sensitivity analysis. Finally, Section 6 provides preliminary conclusions and future steps.

\section{VIRTUAL ORGANIZATION INHERITANCE}

VOs, by nature, have temporary and distributed behaviors and are logically and legally dismantled only after the contractual clauses have been all fulfilled. It means that the VO duration can vary from days to years. There are three basic difficulties that make the process of managing VO inheritance - VO inheritance management (VO-I-M) - complex:

- The amount of information of every VO uses to be naturally huge.

- It is difficult to elect which and how relevant information and lessons (successful and unsuccessful) are, as well as how they should be get, interpreted, analyzed, filtered, modeled, stored, organized, contextualized, systemized and showed to managers for more effective decision-making.

- The knowledge about VOs is usually spread among the VOs' members, i.e. each actor knows about what happened with a given VO inside his/her own company, but rarely or insufficiently knows what happened in other VO members.

Few significant results that face these difficulties can be found in the literature, meaning that there are several questions not well covered yet. Actually, it is a direct consequence of the fact that research in the VO-I area is still in its beginning. 
Franke (Franke, 2000) describes a VO from an inter-organizational perspective where the concept of Virtual Web/Corporation as a partnership model is introduced. The basic idea is that virtual web members should make their resource base available to their partners. The knowledge-based perspective brings the potential to build core know-how and therefore to gain competitive advantage. Franke also states that one agent should act as a caretaker during the VO dissolution phase. The importance of the storage of acquired and generated knowledge was emphasized; however none guideline of how to proceed and/or store such knowledge was provided.

According to Blecker (2000), the knowledge management in VOs means designing, controlling, and developing a purpose-oriented knowledge together with the partners. It leads to the union of the knowledge among the VO's participants. Blecker argues that in short term forms of VOs, only the outcome of production process rather than knowledge is shared. This statement is not true when VOs emerge from VBEs that guarantee preparedness and long term partnership.

The approach presented in this paper aims at gathering the information and the knowledge throughout the CNO (for both short-term and long-term VOs) and organizing them in order to make this knowledge easily accessible.

The need related to the knowledge storage in VOs leads to a specific approach, namely VO-I. The authors define it as the set of information and knowledge accumulated from past and current VOs along their entire life cycle. VO-I-M corresponds to the $\mathrm{VO}$ activity that manages what has been inherited about given VOs, usually supported by computer systems ${ }^{1}$.

VO inheritance is seen as a horizontal process with the aim of offering "services" to other "clients", like VBE and VO management (VOM) actors and related supporting systems. Figure 1 illustrates the VO-I process. In the bottom it is possible to visualize the whole CNO life-cycle comprising VBE and VO life-cycle. On the top of the figure it is represented the process of information gathering and its transformation into knowledge. The top-left side represents databases storing information from VBE partners, VO's performance metrics, clients, products, core competences, and whatever is important. Those databases are one of the sources of information for extracting knowledge (via data mining, for example). In a similar way, on the top-right side the unorganized information is represented by small circles. In an upper level the information is organized according to some rules or relevance; new knowledge - circles in bold - is also made available. For instance, the transformation of tacit knowledge into explicit knowledge via decision trees, questionnaires, and so on. The double-arrows in the middle of the figure represent the data/information flowing from $\mathrm{CNO}$ level to VO-I to be organized and processed, as well as its return to $\mathrm{VBE} / \mathrm{VO}$. The overall process of gathering, storing, organizing, and extracting knowledge from information, related to CNOs, comprises the VOI process.

\footnotetext{
${ }^{1}$ VO-I-M is much more than Business Intelligence (BI). Although some works (e.g. Rabelo et al., 2002) have extended the traditional view of BI when also considering current/dynamic information of a company, BI tools work only with static and past information from just a given company, and not from (dynamic) VOs or supply chains, for instance.
} 


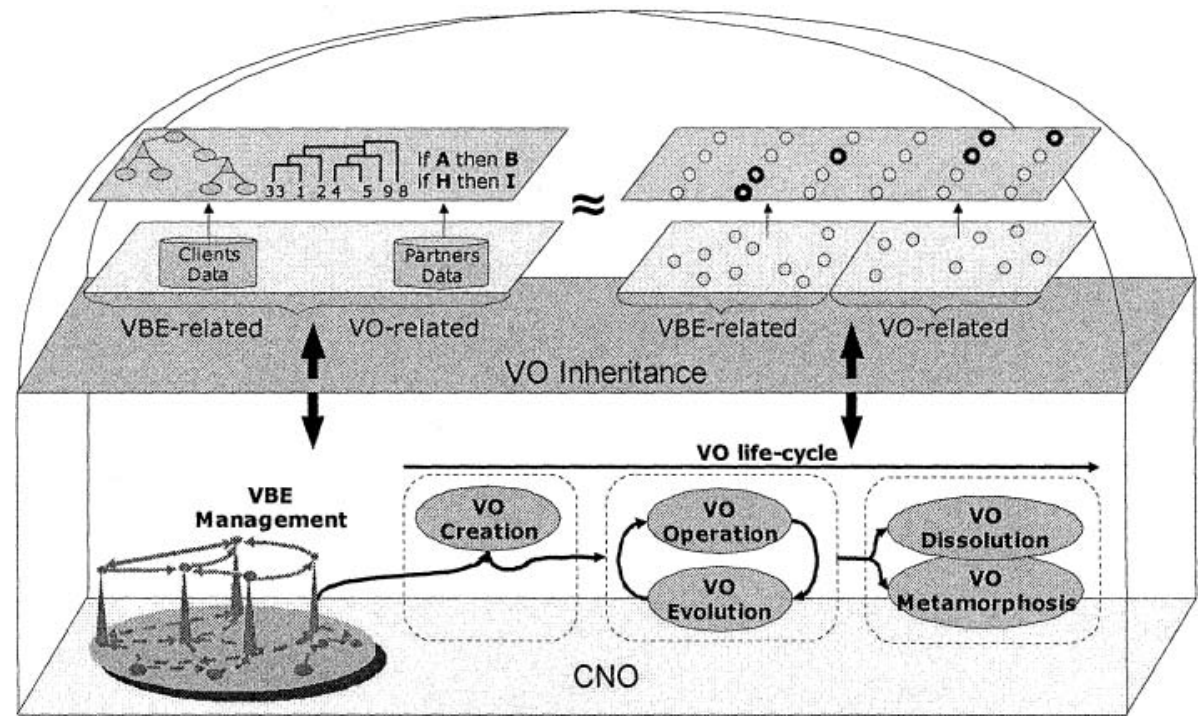

Figure 1 - Global framework for VO inheritance

Finally it is important to differentiate VO inheritance from VO life history. The VO life history is related to the time sequence of actions and their description in the stages during the VO life cycle (Tølle, 2002). As there is not a standard way in which activities can be performed over time, each VO has its own integrated activities describing its life history. Examples of life history can be found in Ollus (2002) and Tølle (2002). In VO-I knowledge and the expertise acquired can also be reused in VOs in execution. For example, comparing partner's performance in different VOs, or predicting cases when planned activities will have some delay. In its essence, VO inheritance aims at improving VO managers' decision making capability. One supporting approach for that is knowledge management.

\section{PROPOSED KNOWLEDGE MANAGEMENT APPROACH}

The definition for Knowledge Management presented by Malhotra (1998) states that "knowledge management caters to the critical issues of organizational adaptation, survival and competence in face of increasingly discontinuous environmental change. Essentially, it embodies organizational processes that seek synergistic combination of data and information processing capacity of information technologies, and the creative and innovative capacity of human beings". Based on this, knowledge management applied to VO inheritance intends to create the synergy among pieces of information. In general, VO inheritance activity collects data and information by itself and it can discover new knowledge (e.g. Loss et al., 2005), whereas knowledge management organizes overall knowledge and makes it available to users in a structured and comprehensive way.

Some suggested tools/techniques for the task of knowledge discovery are: data mining algorithms (Fayyad, 1996) - for finding new patterns and/or models in data 
which can be useful; text and web mining (Mladenic, 2003) - for analyzing and discovering knowledge in unstructured data (e.g. documents in free text form); and decision analysis (Bohanec, 2003) - for deciding the best choice among a number of alternatives.

For the task of organizing and presenting knowledge, knowledge map is an interesting alternative because it corresponds to a visual representation of a knowledge domain in order to facilitate the location, comprehension or development of knowledge (Plumley, 2003). It deals with: i) explicit knowledge - core competencies, legacy systems, additional expertise, forms, documents, presentations, papers; ii) tacit knowledge - group dynamics, culture and social attributes; and iii) procedures (guidance, tips, checklists) - used by the CNO actors.

VO inheritance linked to knowledge management is more than a sum of parts of data and knowledge. It provides a framework with experiences and case studies for managers of both VBEs and VOs. Next section describes the general approach on how a decision support environment can be used as an integrated framework for handling the knowledge extracted in a VO inheritance scenario.

\section{DECISION SUPPORT IN VO INHERITANCE}

Decision Support is a broad term that comprehends all aspects related to supporting people in decision-making. Similarly to what happens in traditional companies, decision making problems are seen as the dilemma of choices also in VO where optimality rarely exist. That is why terms like "the best" or equivalent does not make sense anymore to be applied in real business regarding the so many variables and uncertainties usually presented in a decision. A VO manager is always confronted to situations like finding partners for a VO, evaluating current versus planned scheduling, analyzing financial effects of order replenishment or cancellation, comparing VOs' historical data for performance analysis, planning actions for handling predicted problems, among others. All this aims to fulfill the VOs' goals and contractual clauses regarding expected benefits and profits.

Therefore, in general, the relevant question is "which alternative should be chosen when the VO manager has many variables involved to take a decision?", which reveals making a choice out of a number of alternatives (Bohanec, 2003).

Regarding the field of decision sciences (human decision making), some techniques are available to help in finding options to satisfy the decision maker's goals. The three main branches for decision support are (Bohanec, 2003): i) operation research; ii) decision support systems; and iii) decision analysis (Figure 2). Operation research structures real-life situations into mathematical models. Decision support systems deal with unstructured problems and uses data models to identify and to solve problems. Decision analysis breaks the problem in more manageable parts considering possible alternatives and available information. This paper exploits the use of decision analysis as it seems more suitable for dealing with the mentioned VO problems, which involve uncertainties and managers' preferences.

The process of making choices is more complex than just picking one option. It consists in assessing the current problem and understanding the objectives, and collecting as well as verifying available and pertinent information in order to identify possible alternatives (Clemen 1996). Alternatives are split into more 
manageable and understandable parts in order to model the problem and they are presented to the VO manager, who decides to apply one of them or not, also considering that different managers present different behaviors and adopt different management strategies for the same problem (Pereira-Klen et al., 2005).

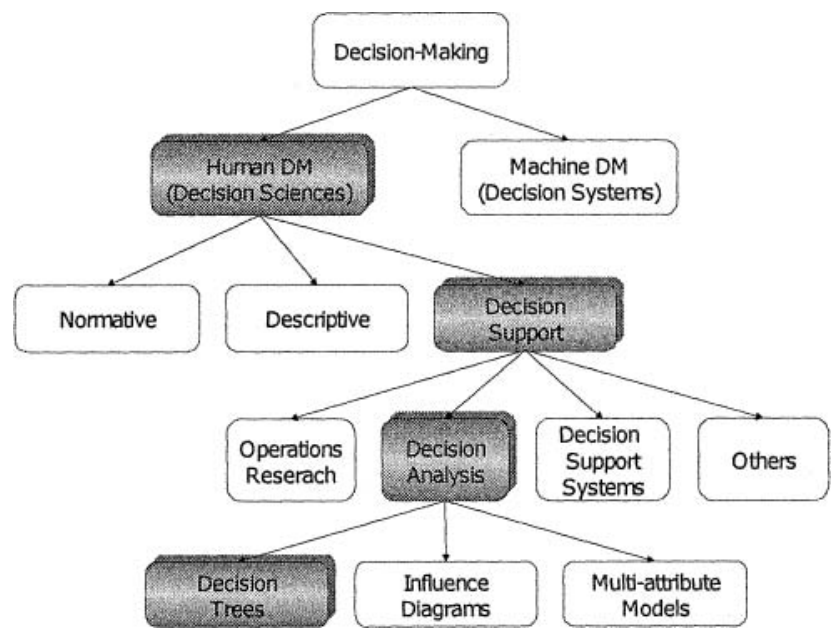

Figure 2 - Decision Making Areas (Source: Clemen, 1996)

Decision trees, a special technique in decision analysis was chosen since they are representations of decision problems and are used in the process of structuring decision elements like alternatives, uncertain events, and final outcomes (Clemen, 1996) which fits our case example. It utilizes a structure of three types of nodes: i) decision nodes: alternatives presented to the manager; ii) chance nodes: events with certain probability of occurrence; and iii) terminal nodes: the final outcome (payoff) for each leaf in the tree. It means that each problem can have two or more alternative solutions (which can be decomposed in several tree levels), and each alternative has decision nodes. At last, each alternative has a probability to happen ( $100 \%$ for all alternatives of a given problem) and an associated cost.

\section{THE EXPERIMENT}

In a CNO scenario a given enterprise is usually involved in several VOs, and decisions should be taken for each VO and for every problem related to. These decisions are different from each other because each situation is likely unique. The example below describe how a decision making process related to one problem in a given VO would be carried out.

This example is related to a case where one VO partner in a given VO is delayed in its planned delivery. In this case, VO-I aims to store information about the decisions taken and their reasons as well as the final outcome for future use by the VO manager. A hypothetic scenario for a VO producing special chairs - named as chairs production - has been devised and decision trees and sensitivity analysis were used to model and to base the final decision for facing such delay. 
In this scenario it was assumed that the foreseen VO profit will be of $10 \mathrm{~K} \in$ in the case no problems occur during its execution. However, as a partner is delayed, a decision should be taken about what to do, regarding other partners' capacities and scheduling, contract penalties, and know-how about the production. It will be also assumed that this problem is considered relevant to be solved (regarding the involved values), its solution should be found as soon as possible (as they affect the current schedule), it will be a one-stage solution (i.e. no further possibilities of refinements/renegotiations), and the VO manager will take the decision alone.

There would be four decisions to be taken:

- Increase capacity - the delayed partner should contract more employees in order to accomplish its deadline. But this will cost $4 \mathrm{~K} €$, i.e. the final outcome will be of $6 \mathrm{~K} €$ and this action has $65 \%$ of probability to overcome the delay. In the case this action does not solve the problem the VO should then pay for this. This means that the other $35 \%$ of probability is divided in other possible consequences. In this case, there would be two types of consequences: the worst one is a very drastic image impact, which is expressed as a zero $€$ of profit; or suffer penalties. Three levels of penalties may be applied, which can vary from low, medium and high penalties, causing a loss of $6.5 \mathrm{~K} €$, $7.5 \mathrm{~K} €$ and $9 \mathrm{~K} €$, respectively, and with a probability of $20 \%, 35 \%$ and $45 \%$, respectively, to happen. The probability numbers and, usually, the values associated with the consequences are provided according to the manager experience and managerial style.

- Status quo - it means to leave it as it is. Status quo is divided into two branches: aware and unaware. The former case represents the manager's knowledge about the overall VO schedule as well as the partners' capacity. The manager can decide to do nothing because he knows that one of the partners has been working with a loose schedule meaning that it is able to absorb the delay. This has $70 \%$ of probability to solve the problem and hence to keep with the original profit of $10 \mathrm{~K} €$. The latter case, the manager thinks that the problem will be "naturally" solved along the time by the involved partners. It has $30 \%$ of probability of success but he estimates a loss of $2.5 \mathrm{~K} €$ in the final outcome with the actions that would be taken by the partners.

- More time - the VO manager requires more time to the final client in order to adjust and to end the delayed production order, but this would be possible thanks a discount of $3 \mathrm{~K} €$. If the client accepts this alternative the final outcome will be $7 \mathrm{KE}$ and this acceptance would happen with $65 \%$ of probability. However, there is $35 \%$ of probability that the client does not accept this. Then the manager should decide for analyzing the consequences image damage or penalties, described in the first decision type.

- Sub-contracting - the VO manager decides to go one step beyond and one or more partners will join the VO in order to fulfill the needs. The available options regarding time, costs, and quality of service are: i) partner 1 will cost of $5 \mathrm{KE}$; ii) partner $25.5 \mathrm{KE}$; iii) partner $36 \mathrm{KE}$; and iv) partner 4 also $5 \mathrm{~K} €$. All these subcontracted partners have $90 \%$ of probability to succeed in this mission. In the case of failure (with $10 \%$ of probability), the profit is zero KE.

Rolling back the options of this decision tree and ranking them, the following sequence is found: more time with an outcome of $€ 5.258,75$; sub-contracting with $€$ 
$4.950,00$; status quo with $€ 4.750,00$; and increase capacity (work power) in the last place with an outcome of $€ 4.608,75$.

Quantities incorporated into a tree are uncertain and many probabilities are just estimations. One option to validate the best alternative is to apply the Sensitivity Analysis technique. It is done ranging the values of some probabilities in order to assess how the decision is affected by the variation in one or more of the uncertain quantities in the model. It works like a what-if case. Figure 3 shows for example that the more the manager is aware about the status of the VO's partners the bigger the chances of taking the right decision tends to be. The probability of awareness, variable $p_{-}$awareness, was ranged from $50 \%$ to $90 \%$. The graphic shows a threshold in $73 \%$, which means that only if the VO manager is more than $75 \%$ sure about his actions, "doing nothing" is a better choice than more time. Just to remember, more time was the best outcome before ranging the value of the probability p-awareness. The results sound obvious or too simple. However no one was aware of this conclusion before this analysis.

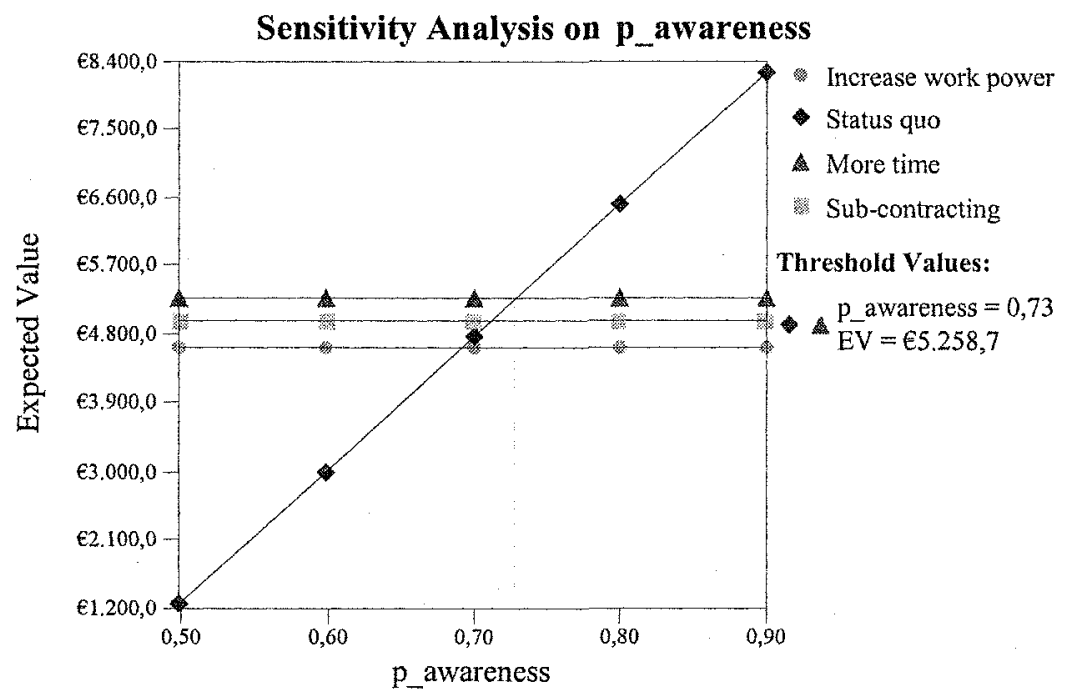

Figure 3 - Sensitivity Analysis on probability of awareness

Despite decision trees cannot be completely reusable regarding that each situation is usually unique, the case presented can be used as a model to be followed in similar situations. Surely different sets of alternatives will be made available in each situation. Sensitivity analysis can be used to study the deviation in some variables representing the probability in chance nodes in order to give more confidence to the decisions. In this context, knowledge management makes the knowledge obtained in decision analysis process explicit and hence easier to be modeled, systemized and available to VO managers for his further use. The content of this decision tree is hierarchically organized and classified in a taxonomy (represented by an ontology) so that short explanations can be provided about the elected decision option (status-quo). 


\section{CONCLUSIONS AND NEXT STEPS}

This paper stressed the concept of Virtual Organization inheritance (VO-I), highlighting its increasing importance in the CNO discipline. An approach for supporting virtual organization inheritance was introduced, where knowledge management was used as the main guideline and decision support was the example chosen for the application presented. The rationale of the proposed approach is that the management of VOs could be much more effective and its problems solved with more agility and reliability if information and lessons learned from past VOs could be stored and properly retrieved by VO actors (e.g. VO managers and VO planners).

Regarding that problems and solutions can vary for every $\mathrm{VO}$, the main goal of VO-I is not necessarily the reusability of decisions taken in the past but rather the possibility to consult what has happened in similar cases, the practices used and their effects, the adequacy of certain performance indicators to select partners to compose a $\mathrm{VO}$ as well as to monitor them along the VO operation, and so forth. Managing VO-I is therefore an important asset for organizations, specially if it is considered that this information is properly stored, processed, and organized. VO inheritance provides structure and guidance for thinking systematically about decisions even the ones that seem to be tricky to solve.

Despite its importance, the research about VO inheritance is still in its beginning and many open questions are present. Some of them were pointed out in the paper, showing an initial research path to this area. This paper did not intend to provide solutions for all the identified problems of decision-making in VOs, but rather to present an initial experiment that corresponds to a part of a wider framework.

In a former work developed the by the authors (Loss, 2005) the focus was put on how bunches of information can be collected and how new knowledge can be generated for decision-making purposes applying data mining. The present work adds another piece in the devised framework evaluating decision trees as a possible technique to support the modeling and costs of decision alternatives. However it is important to point out that the limitation of techniques such as decision tress (for instance the need of experts for both decision tree modeling and domain specific ones) has a direct influence on its application.

Next steps of this research will be devoted to analyze how VO-I can benefit from ontology in terms of better representation of knowledge, richer semantics to link the stored knowledge with problems, and a more sophisticated indexation mechanism to facilitate the search for knowledge by VO managers.

\subsection{Acknowledgments}

This work has been partially supported by the Brazilian councils of research and scientific development - CNPq (www.cnpq.br) and CAPES (www.capes.gov.br). It has been developed in the scope of the Brazilian IFM project (www.ifm.org.br) and the European IST FP-6 IP ECOLEAD project (www.ecolead.org). Special thanks to Mr. Marko Bohanec, from JSI - Slovenia, for his substantial support during the phase of the application of decision analysis techniques, and to Mr. Rui Jorge Tramontin Jr., from UFSC - Brazil, for his valuable comments about the paper. 


\section{REFERENCES}

1. Afsarmanesh, H.; Camarinha-Matos, L.M.; 2005. A Framework for Management of Virtual Organization Breeding Environments. Proceedings PRO-VE'2005, pp 35-48.

2. Blecker, T.; Neumann, R.; 2000. Interorganizational Knowledge Management: Some Perspectives for Knowledge Oriented Strategic Management in Virtual Organizations. In: Malhotra, Y. (ed) Knowledge Management and Virtual Organizations. Idea Group Publishing.

3. Bohanec, M.; 2003. Decision Suppot. In: Mladenic, D.; Lavrac, N.; Bohanec, M.; Moyle, S.; (eds). Data Mining and Decision Support: Integration and Collaboration. Kluwer Academic Publishers - Boston/Dordrecht/London - ISBN 1-4020-7388-8. pp. 23-36.

4. Camarinha-Matos, L. M.; Afsarmanesh, H.; 2004a. Towards Next Business Models. In Collaborative Networked Organizations: a research agenda for emerging business models.

5. Camarinha-Matos, L. M.; Afsarmanesh, $\mathrm{H}_{1} ; 2004 \mathrm{~b}$. Support Infrastructures for New Collaborative Forms. In Collaborative Networked Organizations: a research agenda for emerging business models, Kluwer Academic Publishers, pp. 175-192.

6. Clemen, R.T.; 1996. Making Hard Decisions: An Introd. to Decision Analysis, Duxbury Press.

7. ECOLEAD, 2005a. ECOLEAD Project: European Collaborative Networked Organizations Leadership Initiative. Deliverable D23.1 ECOLEAD Project - Requirements and mechanisms for VO planning and launching.

8. ECOLEAD, 2005b. ECOLEAD Project: European Collaborative Networked Organizations Leadership Initiative. Deliverable D32.2 ECOLEAD Project - Report on Methodologies, Processes and Services for VO Management.

9. ECOLEAD, 2005c. ECOLEAD Project: European Collaborative Networked Organizations Leadership Initiative. Deliverable D21.1 ECOLEAD Project - Characterization of Key Components, Features, and Operating Principles of the VBE.

10. Fayyad, U.; Piatetski-Shapiro, G.; Smyth, P.; 1996. From Data Mining to Knowledge Discovery in Databases. AAAIMIT Press, p.37-54, 1996.

11. Franke, U.; 2000. The Knowledge-Based View of the Virtual Web, the Virtual Coportation, and the Net-Broker. In: Malhotra, Y. (ed) Knowledge Management and Virtual Organizations. Idea Group Publishing.

12. Karvonen, I.; Salkari, I; Ollus, M. ; 2005. Characterizing Virtual Organizations and their Management. Proceedings PRO-VE'2005, pp 193-204.

13. Loss, L.; Rabelo, R. J.; Pereira-Klen, A. A.; 2005. A Generic Framework based on Machine Learning Techniques for Virtual Organization Management. Proc. PRO-VE'2005, pp. 217-226.

14. Malhotra, Y; 1998. Deciphering the Knowledge Management Hype. The Journal for Quality \& Participation, July/August 1998 (Special issue on Knowledge Management), published by the Association for Quality \& Participation.

15. McKenzie, J.; van Winkelen, C.; 2001. Exploring E-collaboration Space. In: The First Annual Knowledge Management Forum Conference. Proceedings. Henley Management College.

16. Mladenic,. D., Grobelnik, M.; 2003. Text and Web Mining. In: Mladenic, D.; Lavrac, N.; Bohanec, M.; Moyle, S.; (eds). Data Mining And Decision Support: Integration and Collaboration. Kluwer Academic Publishers - Boston/Dordrecht/London, pp. 23-36.

17. Ollus, M., Hartel, I., Tølle, M., Wubben, H., Hannus, M. (2002) Using scenarios for dissemination, Experiences from the IMS GLOBEMEN project, Proceedings of eBeW2002 conference, Prague, 16-18 October 2002.

18. Pereira-Klen, A. A.; Klen, E. R.; 2005. Human Supervised Virtual Organization Management. Proceedings PRO-VE'2005, pp 229-238.

19. Plumley, D.; 2003. Process-Based Knowledge Mapping: A practical approach to priorizing knowledge in terms of its relevance to a business or KM objective. Web page accessed in December $20^{\text {th }}, 2005$. URL: (http://www.destinationkm.com/).

20. Rabelo, R. J.; Pereira-Klen, A.; 2002. Business Intelligence Support for Supply Chain Management, Proceedings BASYS'2002 Conference, pp. 437-444.

21. Rabelo, R. J.; Pereira-Klen, A.; Klen, E. R., Effective Management of Dynamic Supply Chains, in International Journal of Networking and Virtual Organizations, 2004.

22. Reed Jr., P. R.; 2002. Reference Architecture: The best of best practices. http:/www128.ibm.com/developerworks/rational/library/2774.html.

23. Tølle, M.; Bernus, P.; Vesterager, J. 2002. Reference models for virtual enterprises. In. Camarinha-Matos, L. M.; Afsarmanesh, H. (Eds). Collaborative Business Ecosystems and Virtual Enterprises. Boston: Kluwer, pp. 3-10. 